Pacific Journal of Mathematics

ON CERTAIN POSER AND SEMILATTICE HOMOMORPHISMS 


\title{
ON CERTAIN POSET AND SEMILATTICE HOMOMORPHISMS
}

\author{
C. S. JoHnson, JR.
}

In this paper a coordinatizing semigroup is used to define and characterize certain homomorphisms on a bounded poset or semilattice. These homomorphisms are determined by their kernels and in the semilattice case the ideals which occur as such kernels are characterized.

1. Introduction. In [4] B. J. Thorne characterized certain congruence relations on a bounded lattice by looking at AP homomorphisms on a coordinatizing Baer semigroup. We intend to carry out a similar procedure for bounded posets and semilattices. It will turn out that one of our semilattice results gives Thorne's central result as a corollary.

Our notation will be that of [4]. If $S$ is a semigroup with 0 and $A \leqq S$ we define $L(A)=\{x \in S ; x a=0$ for all $a \in A\}, R(A)=\{x \in S ; a x=$ 0 for all $a \in A\}, L R(A)=L(R(A)), R L(A)=R(L(A))$, and so forth. If $x \in S$ we write $L(\{x\})=L(x)$ and $R(\{x\})=R(x)$. We define $\mathscr{L}(S)=$ $\{L(x) ; x \in S\}$ and $\mathscr{R}(S)=\{R(x) ; x \in S\}$ and say that $S$ coordinatizes a poset $P$ in case $P \cong \mathscr{L}(S)$ when $\mathscr{L}(S)$ is partially ordered by set inclusion.

The coordinatization machinery which we will use is developed in [2]. The following is a summary of the relevant material.

Definition 1.1. A semigroup $S$ with 0 and 1 will be called a preBaer semigroup in case, for each $x \in S$, there exist elements $x^{r}, x^{l} \in S$ such that $L R(x)=L\left(x^{r}\right)$ and $R L(x)=R\left(x^{l}\right)$.

Recall that a map $\phi$ of a poset $P$ into itself is residuated if the inverse image of a principal ideal is again a principal ideal or, equivalently, if $\phi$ is isotone and there is another isotone map $\phi^{+}$(called a residual map) of $P$ into itself such that $x \phi^{+} \phi \leqq x \leqq x \phi \phi^{+}$for all $x \in P$.

LEMMA 1.2. If $S$ is a pre-Baer semigroup and $z \in S$, then $\phi_{z}: \mathscr{L}(S) \rightarrow \mathscr{L}(S)$ given by $L R(x) \dot{\phi}_{z}=L R(x z)$ is residuated with $\phi_{z}^{+}: \mathscr{L}(S) \rightarrow \mathscr{L}(S)$ given by $L(x) \phi_{z}^{+}=L(z x)$ as its residual.

If $P$ is a bounded poset we use $S(P)$ to denote the semigroup of residuated maps on $P$.

THEOREM 1.3. Every bounded poset can be coordinatized by a pre- 
Baer semigroup. In particular, if $P$ is a bounded poset, then $S(P)$ is a pre-Baer semigroup which coordinatizes $P$. If $S$ is any other preBaer semigroup which coordinatizes $P$, then $z \mapsto \phi_{z}$ is a homomorphism, with kernel 0, of $S$ into $S(P)$ and the image of $S$ in $S(P)$ is a preBaer semigroup which coordinatizes $P$.

DeFinition 1.4. A pre-Baer semigroup $S$ is a right Baer semigroup in case for each $x \in S$ there exists an idempotent $x^{r} \in S$ such that $R(x)=x^{r} S$, i.e., such that $x y=0 \Leftrightarrow y=x^{r} y . \quad S$ is a left Baer semigroup in case for each $x \in S$ there exists an idempotent $x^{l} \in S$ such that $L(x)=S x^{l}$.

THEOREM 1.5. Every right (resp., left) Baer semigroup coordinatizes a bounded join (resp., meet) semilattice. Conversely, every bounded join (resp., meet) semilattice can be coordinatized by a right (resp., left) Baer semigroup. In particular, if $P$ is a bounded join (resp., meet) semilattice, then $S(P)$ is a right (resp., left) Baer semigroup which coordinatizes $P$. If $S$ is any other right (resp., left) Baer semi-group which coordinatizes $P$ then the image of $S$ in $S(P)$ under the homomorphism $z \mapsto \phi_{z}$ is a right (resp., left) Baer semigroup.

REMARK. If $S$ is a right Baer semigroup the join operation in

$(S)$ is given by $L R(x) \vee L R(y)=L\left(y^{r}\left(x y^{r}\right)^{l}\right)=L R(x) \phi_{y^{r}} \phi_{y^{r}}^{+}$. If $S$ is a left Baer semigroup the meet operation in $\mathscr{L}(S)$ is given by $L(x) \cap$ $L(y)=L R\left(\left(y^{l} x\right)^{l} y^{l}\right)=L(x) \phi_{x}^{+} l \phi_{y} l$.

\section{Homomorphisms preserving $r$ and $l$.}

DEFINITION 2.1. A homomorphism $\phi$ of a pre-Baer semigroup $S$ onto a semigroup $T$ is called $r$-preserving in case, for each $x \in S, L R(x \phi)=$ $L\left(x^{r} \phi\right)$ for some choice of $x^{r}$. (Recall $x^{r}$ is such that $L R(x)=L\left(x^{r}\right)$.) $\phi$ is $l$-preserving in case, for each $x \in S, R L(x \phi)=R\left(x^{l} \phi\right)$ for some choice of $x^{l}$. (Recall $x^{l}$ is such that $R L(x)=R\left(x^{l}\right)$.) Notice that if $\phi$ is $r$-and $l$ - preserving, then $T$ is a pre-Baer semigroup.

LEMMA 2.2. Let $\phi$ be a homomorphism of a pre-Baer semigroup $S$ onto a semigroup $T$.

(i) If $\phi$ is r-preserving, then $\Phi: \mathscr{L}(S) \rightarrow \mathscr{L}(T)$ given by $L R(x) \Phi=$ $L R(x \phi)$ is well defined and isotone.

(ii) If $\phi$ is l-preserving, then $\Phi: \mathscr{L}(S) \rightarrow \mathscr{L}(T)$ given by $L(x) \Phi=$ $L(x \phi)$ is well defined and isotone.

Proof. (i). Suppose that $\phi$ is $r$-preserving and that $L R(x) \subseteq L R(y)$. Choose $y^{r}$ so that $L R(y \phi)=L\left(y^{r} \phi\right)$. Then we have $L R(x) \subseteq L R(y) \Rightarrow$ $x \in L R(x) \subseteq L\left(y^{r}\right) \Rightarrow x y^{r}=0 \Rightarrow x \phi y^{r} \phi=0 \Rightarrow x \phi \in L\left(y^{r} \phi\right)=L R(y \phi) \Rightarrow L R(x \phi) \subseteq$ 
$L R(y \phi)$. This shows that $\Phi$ is well defined and isotone. Finally, $L R(x) \Phi=L\left(x^{r} \phi\right) \in \mathscr{L}(T)$.

(ii). Suppose that $\phi$ is $l$-preserving and that $L(x) \subseteq L(y)$. Choose $x^{l}$ so that $R L(x \phi)=R\left(x^{l} \phi\right)$. Then we have $L(x) \leqq L(y) \Rightarrow R L(y) \subseteq$ $R L(x) \Rightarrow y \in R L(y) \subseteq R\left(x^{l}\right) \Rightarrow x^{l} y=0 \Rightarrow x^{l} \phi y \phi=0 \Rightarrow y \phi \in R\left(x^{l} \phi\right)=R L(x \phi) \Rightarrow$ $R L(y \phi) \subseteq R L(x \phi) \Rightarrow L(x \phi) \subseteq L(y \phi)$. This makes $\Phi$ well defined and isotone.

REMARK. Notice that, in part (i) of the lemma, $L(x) \Phi=L R\left(x^{l}\right) \Phi=$ $L R\left(x^{l} \phi\right)$. Hence $L(x) \Phi=L(x \phi)$ for all $x \in S$ iff $\phi$ is $l$-preserving. Similarly, in part (ii), $L R(x) \Phi=L\left(x^{r} \phi\right)$ and it is clear that $L R(x) \Phi=L R(x \phi)$ for all $x \in S$ iff $\phi$ is $r$-preserving. If $\phi$ is $r$-and $l$-preserving, then the mappings in parts (i) and (ii) of the lemma coincide.

If $S$ is a pre-Baer semigroup and $\phi: S \rightarrow T$ (i.e., from $S$ onto $T$ ) an $r$-preserving homomorphism, then the map defined in part (i) of Lemma 2.2 induces an equivalence relation $\equiv$ on $\mathscr{L}(S)$ by the rule $L R(x) \equiv L R(y)$ iff $L R(x) \Phi=L R(y) \Phi$ iff $L R(x \phi)=L R(y \phi)$. It is this equivalence relation we wish to examine.

Definition 2.3. If $S$ is a pre-Baer semigroup and $\phi: S \rightarrow T$ an $r$-preserving homomorphism, then the equivalence relation on $\mathscr{L}(S)$ just described will be called the equivalence relation on $\mathscr{L}(S)$ induced by $\phi$.

Definition 2.4. An equivalence relation $\equiv$ on $\mathscr{L}(S)$ where $S$ is a pre-Baer semigroup is $S$-compatible in case $L R(x) \equiv L R(y) \Longrightarrow L R(x) \phi_{z} \equiv$ $L R(y) \phi_{z}$ for all $z \in S$. It is $S^{+}$-compatible in case $L R(x) \equiv L R(y) \Rightarrow$ $L R(x) \phi_{z}^{+} \equiv L R(y) \phi_{z}^{+}$for all $z \in S$.

Definition 2.5. An equivalence relation $\equiv$ on a poset $P$ is ordered if $P / \equiv$ is partially ordered by the rule $[x] \leqq[y] \Leftrightarrow$ there exist elements $x_{1} \in[x]$ and $y_{1} \in[y]$ such that $x_{1} \leqq y_{1}$.

REMARK. Congruence relations on lattices and semilattices are ordered.

LeMMA 2.6. If $\equiv$ is an equivalence relation on $\mathscr{L}(S), S$ a preBaer semigroup, and $\mathscr{L}(S) / \equiv$ is partially ordered in such a way that $L R(x) \leqq L R(y) \Longrightarrow[L R(x)] \leqq[L R(y)]$, then the following are equivalent.

(a) $\left[L R(x) \phi_{z^{r}}\right]=[0] \Rightarrow[L R(x)] \leqq\left[0 \phi_{z^{r}}^{+}\right]$, for all $x \in S$.

(b) $[L R(x)]=[0] \Longrightarrow\left[L R(x) \phi_{z^{+}}^{+}\right]=\left[0 \phi_{z_{r}^{+}}^{+}\right]$, for all $x \in S$.

(b') $L R(x) \equiv 0 \Rightarrow L R(x) \phi_{z^{r}}^{+} \equiv 0 \phi_{z^{r}}^{+}=L R(z)$, for all $x \in S$.

Proof. $(b) \Leftrightarrow\left(b^{\prime}\right)$. This is only a difference in notation.

(a) $\Rightarrow(\mathrm{b})$. Suppose $[L R(x)]=[0]$. Since $L R(x) \phi_{z^{r}}^{+} \phi_{z^{r}} \subseteq L R(x)$, we 
have $\left[L R(x) \phi_{z^{r}}^{+} \phi_{z^{r}}\right]=[0]$. Now by (a), $\left[L R(x) \phi_{z^{r}}^{+}\right] \leqq\left[0 \phi_{z^{r}}^{+}\right]$. The reverse inequality holds since $0 \phi_{z^{r}}^{+} \subseteq L R(x) \phi_{z^{r}}^{+}$.

(b) $\Rightarrow$ (a). If $\left[L R(x) \phi_{z^{r}}\right]=[0]$, we have by (b) that $\left[L R(x) \phi_{z^{r}} \phi_{z^{r}}^{+}\right]=$ $\left[0 \phi_{z^{r}}^{+}\right]$. Now $L R(x) \leqq L R(x) \phi_{z^{r}} \phi_{z^{r}}^{+}$gives $[L R(x)] \leqq\left[L R(x) \phi_{z^{r}} \phi_{z^{r}}^{+}\right]=\left[0 \phi_{z^{r}}^{+}\right]$.

THEOREM 2.7. If $S$ is a pre-Baer semigroup and $\phi: S \rightarrow T$ an $r$ preserving homomorphism, the equivalence relation $\equiv$ on $\mathscr{L}(S)$ induced by $\phi$ has the following properties:

(i) For each $z \in S, z^{r}$ can be chosen so that $L R(x) \equiv 0 \Rightarrow L R(x) \phi_{z^{r}}^{+} \equiv$ $0 \phi_{z^{r}}^{+}$for all $x \in S$.

(ii) $\equiv$ is ordered.

(iii) $\equiv$ is S-compatible.

In part (i ) any $z^{r}$ such that $L\left(z^{r} \phi\right)=L R(z \phi)$ suffices.

Proof. Recall that $L R(x) \equiv L R(y) \Leftrightarrow L R(x \phi)=L R(y \phi)$.

(i ). $\mathscr{L}(S) / \equiv$ is partially ordered by $[L R(x)] \leqq[L R(y)] \Leftrightarrow L R(x \phi) \leqq$ $L R(y \phi)$. Choose $z^{r}$ so that $L\left(z^{r} \phi\right)=L R(z \phi)$. Since $L R(x) \subseteq L R(y) \Rightarrow$ $L R(x \phi) \cong L R(y \phi)$ by Lemma 2.2, we can apply Lemma 2.6. Since $L R(x) \phi_{z^{r}} \equiv 0 \Rightarrow L R\left(x z^{r} \phi\right)=0 \Rightarrow x \phi z^{r} \phi=0 \Rightarrow x \phi \in L\left(z^{r} \phi\right) \Rightarrow L R(x \phi) \leqq L R(z \phi)$ for all $x \in S$, part (a) of Lemma 2.6 is satisfied and part (b) is what we are trying to prove.

(ii). It will suffice to show that $L R(x \phi) \subseteq L R(y \phi) \Rightarrow$ there exists $y_{1} \in S$ such that $L R(x) \subseteq L R\left(y_{1}\right)$ and $L R\left(y_{1} \phi\right)=L R(y \phi)$. If $L R(x \phi) \cong$ $L R(y \phi)=L\left(y^{r} \phi\right)$, we have $x \phi y^{r} \phi=0 \Rightarrow L R\left(x y^{r} \phi\right)=0 \Rightarrow L R\left(x y^{r}\right)=0$. By (i), $L R\left(x y^{r}\right) \phi_{y^{r}}^{+} \equiv 0 \phi_{y^{r}}^{+}=L R(y)$. Since $L R\left(x y^{r}\right) \phi_{y^{r}}^{+}=L\left(y^{r}\left(x y^{r}\right)^{r}\right)=$ $L R\left(\left(y^{r}\left(x y^{r}\right)^{r}\right)^{l}\right)$, this says that $L R\left(\left(y^{r}\left(x y^{r}\right)^{r}\right)^{l} \phi\right)=L R(y \phi)$. Letting $y_{1}=$ $\left(y^{r}\left(x y^{r}\right)^{r}\right)^{l}$ finishes the proof since $x \in L\left(y^{r}\left(x y^{r}\right)^{r}\right) \Rightarrow L R(x) \leqq L\left(y^{r}\left(x y^{r}\right)^{r}\right)=$ $L R\left(\left(y^{r}\left(x y^{r}\right)^{r}\right)^{l}\right)=L R\left(y_{1}\right)$.

(iii). $\quad L R(x) \equiv L R(y) \Rightarrow L R(x \phi)=L R(y \phi) \Rightarrow L R(x \phi z \phi)=L R(y \phi z \phi) \Rightarrow$ $L R(x z \phi)=L R(y z \phi) \Rightarrow L R(x) \phi_{z} \equiv L R(y) \phi_{z}$.

The equivalence relation in Theorem 2.7 has another nice property. It is determined by its kernel.

THeOREM 2.8. Let $\equiv$ be the equivalence relation of Theorem 2.7. The following are equivalent.

(a) $L R(x) \equiv L R(y)$.

(b) If $L\left(x^{r} \phi\right)=L R(x \phi)$ and $L\left(y^{r} \phi\right)=L R(y \phi)$, then $L R(x) \phi_{y^{r}} \equiv 0$ and $L R(y) \phi_{x^{r}} \equiv 0$.

Proof. $\quad(\mathrm{a}) \Rightarrow(\mathrm{b}) . \quad$ Since $\equiv$ is $S$-compatible, $L R(x) \equiv L R(y) \Rightarrow$ $L R(x) \phi_{y^{r}} \equiv L R(y) \phi_{y^{r}}=0$. Similarly $L R(y) \phi_{x^{r}} \equiv 0$.

(b) $\Rightarrow$ (a). Part (b) of Lemma 2.6 is satisfied by Theorem 2.7, so by part (a) of Lemma 2.6, $L R(x) \phi_{y^{r}} \equiv 0 \Rightarrow[L R(x)] \leqq\left[0 \phi_{y^{r}}^{+}\right]=[L R(y)]$. Similarly $L R(y) \phi_{x^{r}} \equiv 0 \Rightarrow[L R(y)] \leqq[L R(x)]$. Thus $[L R(x)]=[L R(y)]$. 
We now wish to show that any equivalence relation on $\mathscr{L}(S)$ having the three properties of Theorem 2.7 is induced on $\mathscr{L}(S)$ by some $r$-preserving homomorphism.

LEMmA 2.9. Let $S$ be a pre-Baer semigroup and let $\equiv$ be an S-compatible equivalence relation on $\mathscr{L}(S)$. For each $z \in S$ define $\Phi_{z}: \mathscr{L}(S) / \equiv$ $\rightarrow \mathscr{L}(S) / \equiv b y[L R(x)] \Phi_{z}=\left[L R(x) \phi_{z}\right]=[L R(x z)] . \quad \Phi_{z}$ is well defined because of S-compatibility. Let $S^{\prime}$ denote the semigroup generated by $\left\{\Phi_{z} ; z \in S\right\}$ under composition. The map $z \mapsto \Phi_{z}$ is a homomorphism of $S$ onto $S^{\prime}$ and if $\equiv$ also possesses properties (i) and (ii) of Theorem 2.7, this homomorphism is r-preserving.

Proof. It is a clear that $z \mapsto \Phi_{z}$ is a homomorphism of $S$ onto $S^{\prime}$. Let $z \in S$ and choose $z^{r}$ to satisfy part (i) of Theorem 2.7. $\Phi_{z} \Phi_{z^{r}}=0$ since $z z^{r}=0$ so we have $L R\left(\Phi_{z}\right) \subseteq L\left(\Phi_{z^{r}}\right)$. To show that $L\left(\Phi_{z^{r}}\right) \subseteq L R\left(\Phi_{z}\right)$ we suppose that $\Phi_{x} \in L\left(\Phi_{z}\right)$ and show that $\Phi_{y} \in R\left(\Phi_{z}\right)$ implies $\Phi_{x} \Phi_{y}=0$. Since $\Phi_{x z^{r}}=0$ we have $[L R(1)] \Phi_{x z^{r}}=\left[L R\left(x z^{r}\right)\right]=0$ and by Lemma 2.6, which applies since we are assuming part (i) of Theorem $2.7,[L R(x)] \leqq$ $[L R(z)]$. Since $\equiv$ is ordered, the elements of $S^{\prime}$ are isotone maps and we have $[L R(x y)]=[L R(x)] \Phi_{y} \leqq[L R(z)] \Phi_{y}=[L R(z y)]=[L R(1)] \Phi_{z y}=$ [0]. Now $[L R(1)] \Phi_{x y}=[0]$ implies $\Phi_{x y}=\Phi_{x} \Phi_{y}=0$.

REMARK. If an S-compatible equivalence relation $\equiv$ possesses properties (i) and (ii) of Theorem 2.7, and if we denote the kernel of $z \mapsto \Phi_{z}$ by $I$, then $z \mapsto \Phi_{z}$ is the homomorphism studied by R. S. Pierce in [3]. To prove this we must show that $\Phi_{x}=\Phi_{y} \Leftrightarrow a x b \in I$ iff $a y b \in I$. Suppose $\Phi_{x}=\Phi_{y}$. Then $a x b \in I \Leftrightarrow \Phi_{a x b}=\Phi_{a} \Phi_{x} \Phi_{b}=0 \Leftrightarrow \Phi_{a y b}=\Phi_{a} \Phi_{y} \Phi_{b}=$ $0 \Leftrightarrow a y b \in I$. Now suppose $a x b \in I$ iff, $a y b \in I$. Then $\Phi_{z x w}=0$ iff $\Phi_{z y w}=$ $0 \Rightarrow[L R(z x w)]=[0]$ iff $[L R(z y w)]=[0] \Rightarrow\left[L R(z x) \phi_{w}\right]=[0]$ iff $\left[L R(z y) \Phi_{w}\right]=$ [0]. Setting $w=(z x)^{r}$, where $(z x)^{r}$ is chosen as in part (i ) of Theorem 2.7, and using part (a) of Lemma 2.6 we have $[L R(z y)] \leqq\left[L\left((z x)^{r}\right)\right]=$ $[L R(z x)]$. Similarly we have $[L R(z x)] \leqq[L R(z y)]$. Thus $[L R(z x)]=$ $[L R(z y)]$ for all $z \in S$, but this just says that $\Phi_{x}=\Phi_{y}$.

THEOREM 2.10. Let $S$ be a pre-Baer semigroup and let $\equiv$ be an equivalence relation on $\mathscr{L}(S)$ which possesses properties (i), (ii), and (iii) of Theorem 2.7. Then $\equiv$ is induced on $\mathscr{L}(S)$ by the r-preserving homomorphism $z \mapsto \Phi_{z}$ described in Lemma 2.9. Furthermore, $z \mapsto \Phi_{z}$ is the largest r-preserving homomorphism (considered as a congruence relation on $S$ ) which induces $\equiv$.

Proof. Consider the $r$-preserving homomorphism $z \mapsto \Phi_{z}$ of Lemma 2.9. We wish to show that $L R\left(\Phi_{x}\right)=L R\left(\Phi_{y}\right)$ iff $L R(x) \equiv L R(y)$. Let $L R\left(\Phi_{x}\right)=L R\left(\Phi_{y}\right)$ and choose $y^{r}$ as in part (i ) of Theorem 2.7. Then 
$R\left(\Phi_{x}\right)=R\left(\Phi_{y}\right)$ and we have $\Phi_{x} \Phi_{y^{r}}=0$ since $\Phi_{y} \Phi_{y^{r}}=0 . \quad \Phi_{x y}=0$ means $\left[L R\left(x^{r} y\right)\right]=[0]$ and by Lemma $2.6[L R(x)] \leqq[L R(y)]$. Similarly we get $[L R(y)] \leqq[L R(x)]$ and thus $L R(x) \equiv L R(y)$. Conversely, suppose $L R(x) \equiv L R(y)$. Choose $x^{r}$ and $y^{r}$ such that $L\left(\Phi_{x r}\right)=L R\left(\Phi_{x}\right)$ and $L\left(\Phi_{y^{r}}\right) \equiv$ $L R\left(\Phi_{y}\right)$. By $S$-compatibility we have $L R\left(x y^{r}\right) \equiv L R\left(y y^{r}\right)=0$ and $L R\left(y x^{r}\right) \equiv$ $L R\left(x x^{r}\right)=0$. This means $\Phi_{x y}=\Phi_{y x^{r}}=0$. Now $\Phi_{x} \in L\left(\Phi_{y r}\right)=L R\left(\Phi_{y}\right)$ gives $L R\left(\Phi_{x}\right) \subseteq L R\left(\Phi_{y}\right)$ and $\Phi_{y} \in L\left(\Phi_{x^{r}}\right)=L R\left(\Phi_{x}\right)$ gives $L R\left(\Phi_{y}\right) \subseteq L R\left(\Phi_{x}\right)$.

Finally, suppose $\phi$ is another $r$-preserving homomorphism which induces $\equiv$. Then $x \phi=y \phi \Rightarrow z x \phi=z y \phi$ for all $z \in S \Rightarrow L R(z x \phi)=L R(z y \phi)$ for all $z \in S \Rightarrow L R(z) \phi_{x} \equiv L R(z) \phi_{y}$ for all $z \in S \Rightarrow \Phi_{x}=\Phi_{y}$.

REMARK. The $r$-preserving homomorphisms which induce $\equiv$ all have the same kernel since, if $\phi$ is such a homomorphism, $x \phi=0 \Leftrightarrow$ $L R(x \phi)=0 \Leftrightarrow L R(x) \equiv 0$.

THEOREM 2.11. Let $S$ be a pre-Baer semigroup and $\phi: S \rightarrow T$ an $r$-preserving homomorphism. Let $\Phi: \mathscr{L}(S) \rightarrow \mathscr{L}(T)$ be the map described in Lemma 2.2 ( i ), i.e., $L R(x) \Phi=L R(x \phi)$. The following are equivalent.

(a) $\operatorname{ker} \phi \in \mathscr{L}(S)$.

(b) $\operatorname{ker} \Phi$ is a principal ideal.

(c) $\Phi: \mathscr{L}(S) \rightarrow \mathscr{L}(T)$ is residuated.

Proof. (a) $\Leftrightarrow$ (b). This follows from the observation that $x \in \operatorname{ker} \phi \Leftrightarrow$ $x \phi=0 \Leftrightarrow L R(x \phi)=0 \Leftrightarrow L R(x) \in \operatorname{ker} \Phi$.

$(\mathrm{c}) \Rightarrow(\mathrm{b})$. This is clear.

$(\mathrm{a}) \Rightarrow$ (c). Suppose $\operatorname{ker} \phi=L R(w)$. Define $\Phi^{+}: \mathscr{L}(T) \rightarrow \mathscr{L}(S)$ by $L(x \phi) \Phi^{+}=L\left(x w^{r}\right) . \quad \Phi^{+}$is well defined and isotone since when $L(x \phi) \leqq$ $L(y \phi)$ we have $z \in L\left(x w^{r}\right) \Rightarrow z x w^{r}=0 \Rightarrow z x \in \operatorname{ker} \phi \Rightarrow z \phi x \phi=0 \Rightarrow z \phi \in L(x \phi) \subseteq$ $L(y \phi) \Rightarrow z \phi y \phi=0 \Rightarrow z y \in \operatorname{ker} \phi \Rightarrow z y w^{r}=0 \Rightarrow z \in L\left(y w^{r}\right)$, which says that $L\left(x w^{r}\right) \leqq L\left(y w^{r}\right)$. Choose $x^{r}$ so that $L\left(x^{r} \dot{\phi}\right)=L R(x \phi)$. Now since $x \in$ $L\left(x^{r} w^{r}\right)$ we have $L R(x) \subseteq L\left(x^{r} w^{r}\right)=L\left(x^{r} \phi\right) \Phi^{+}=L R(x \phi) \Phi^{+}=L R(x) \Phi \Phi^{+}$. Now all that remains is to show that $L(x \phi) \Phi^{+} \Phi \subseteq L(x \dot{\phi})$. Since $\left(x w^{r}\right)^{l} x w^{r}=0 \Rightarrow\left(x w^{r}\right)^{l} x \in \operatorname{ker} \phi \Rightarrow\left(x w^{r}\right)^{l} \phi x \phi=0 \Rightarrow\left(x w^{r}\right)^{l} \phi \in L(x \phi)$ we have $L(x \phi) \Phi^{+} \Phi=L\left(x w^{r}\right) \Phi=L R\left(\left(x w^{r}\right)^{l} \phi\right) \leqq L(x \phi)$.

If $S$ is a pre-Baer semigroup and $z \in S$, notice that $\mathscr{R}(S)$ is dual isomorphic to $\mathscr{L}(S)$ and the residuated map on $\mathscr{R}(S)$ given by $R L(x) \mapsto R L(z x)$, considered as a map on $\mathscr{L}(S)$, is $\phi_{z}^{+}$. (See Lemma 1.2.) Bearing this in mind and applying left-right duality to the results obtained thus far, we find that every $l$-preserving homomorphism on a pre-Baer semigroup $S$ induces on $\mathscr{L}(S)$ an ordered $S^{+}$-compatible equivalence relation $\equiv$ with the property that, for each $z \in S, z^{l}$ can be chosen so that $L R(x) \equiv 1 \Rightarrow L R(x) \phi_{y^{l}} \equiv 1 \phi_{z^{l}}$ for all $x \in S$. Further- 
more, every such equivalence relation on $\mathscr{L}(S)$ is induced by some $l$-preserving homomorphism on $S$. We now have

THEOREM 2.12. Let $\phi$ be an $r$-and l-preserving homomorphism on a pre-Baer semigroup $S$. The ordered equivalence relation on $\mathscr{L}(S)$ induced by $\phi$ is $S$ - and $S^{+}$-compatible. Furthermore, every $S$ - and $S^{+}$compatible ordered equivalence relation on $\mathscr{L}(S)$ is induced by some $r$ - and l-preserving homomorphism on $S$.

Proof. This follows from previous results and the remarks preceding the theorem if we make the following observation: If an ordered equivalence relation $\equiv$ on $\mathscr{L}(S)$ is $S$ - and $S^{+}$-compatible, then $\Phi_{z}: \mathscr{L}(S) / \equiv \Rightarrow \mathscr{L}(S) / \equiv$ given by $[L R(x)] \Phi_{z}=\left[L R(x) \phi_{z}\right]$ is residuated with residual $\Phi_{z}^{+}: \mathscr{L}(S) / \equiv \Longrightarrow \mathscr{L}(S) / \equiv$ given by $[L R(x)] \Phi_{z}^{+}=\left[L R(x) \Phi_{z}^{+}\right]$. Since residuated maps uniquely determine their residuals and vice versa, the $r$-preserving homomorphism $z \mapsto \Phi_{z}$ (considered as a congruence on $S$ ) coincides with the $l$-preserving congruence on $S$ associated with the anti-homomorphism $z \mapsto \Phi_{z}^{+}$.

\section{RAP and LAP homomorphisms.}

DEFinition 3.1. If $S$ is a right Baer semigroup, a semigroup homomorphism $\phi: S \rightarrow T$ is right annihilator preserving or $R A P$ in case $R(x \phi)=R(x) \phi$. Notice that $R(x) \phi=\left(x^{r} \phi\right) T$. Dually, if $S$ is a left Baer semigroup, $\phi$ is left annihilator preserving or $L A P$ in case $L(x \phi)=$ $L(x) \phi$. Finally, $\phi$ is annihilator preserving or $A P$ if it is both $R A P$ and $L A P$.

REMARK. Any $R A P$ homomorphism is $r$-preserving since $L R(x \phi)=$ $L\left(\left(x^{r} \phi\right) T\right)=L\left(x^{r} \phi\right)$. Dually, any $L A P$ homomorphism is $l$-preserving.

LEMMA 3.2. In a right Baer semigroup $S$ we have

(i) $L R(x) \vee L R(x) \phi_{y^{r}} \vee L R(y) \phi_{x^{r}}=L R(y) \vee L R(x) \phi_{y^{r}} \vee L R(y) \phi_{x^{r}}$.

(ii) $L R(z y) \vee L R(x y)=L R\left(z x^{r} y\right) \vee L R(x y)$.

(iii) $L R(x) \vee L R(y) \vee L R\left(x y^{r}\right)=L R(y) \vee L R\left(x y^{r}\right)$.

Proof. It is shown in [2] that, in a right Baer semigroup $S, R(x) \cap$ $R(y) \in \mathscr{R}(S)$ and that the join operation in $\mathscr{L}(S)$ is given by $L R(x) \vee$ $L R(y)=L(R(x) \cap R(y))$.

(i). It is enough to show that $R(x) \cap R\left(x y^{r}\right) \cap R\left(y x^{r}\right)=R(y) \cap$ $R\left(x y^{r}\right) \cap R\left(y x^{r}\right)$. If $z \in R(x) \cap R\left(x y^{r}\right) \cap R\left(y x^{r}\right)$, then $z=x^{r} z$ and $y z=$ $y x^{r} z=0$ so $z \in R(y) \cap R\left(x y^{r}\right) \cap R\left(y x^{r}\right)$. The other inclusion follows by symmetry.

(ii). It is enough to show $R(z y) \cap R(x y)=R\left(z x^{r} y\right) \cap R(x y)$. This 
follows from the observation that if $x y w=0$, then $y w=x^{r} y w$ so that $z y w=0 \Leftrightarrow z x^{r} y w=0$.

(iii). It is enough to show that $R(y) \cap R(x y) \subseteq R(x) \cap R(y) \cap R\left(x y^{r}\right)$. If $y w=0$, then $w=y^{r} w$ so that $x y^{r} w=0 \Rightarrow x w=0$.

LEMMA 3.3. If $S$ is a right Baer semigroup and $\equiv$ is an S-compatible equivalence relation on $\mathscr{L}(S)$, the following are equivalent.

(a) $\equiv$ is a join congruence.

(b) $L R(x) \vee L R(z)=L R(y) \vee L R(z), L R(z) \equiv 0 \Rightarrow L R(x) \equiv L R(y)$.

Proof. $\quad(\mathrm{a}) \Longrightarrow(\mathrm{b})$. Since $L R(z) \equiv 0$, we have $L R(x)=L R(x) \vee 0 \equiv$ $L R(x) \vee L R(z)=L R(y) \vee L R(z) \equiv L R(y) \vee 0=L R(y)$.

(b) $\Rightarrow$ (a). Suppose $L R(x) \equiv L R(y)$. If $L R(z) \in \mathscr{L}(S)$, we have, using Lemma 3.2, that $L R(x) \vee L R(z) \vee L R(x) \phi_{y^{r}} \vee L R(y) \dot{\phi}_{x^{r}}=L R(y) \vee$ $L R(z) \vee L R(x) \phi_{y^{r}} \vee L R(y) \phi_{x^{r}}$. To show that $L R(x) \vee L R(z) \equiv L R(y) \vee$ $L R(z)$ it will suffice, by (b), to show $L R(x) \phi_{y^{r}} \vee L R(y) \phi_{x^{r}} \equiv 0$. Since $\equiv$ is $S$-compatible we have $L R(x) \phi_{y^{r}} \equiv L R(y) \phi_{y^{r}}=0=L R(x) \phi_{x^{r}} \equiv L R(y) \phi_{x^{r}}$. Using (b), $L R(x) \phi_{y^{r}} \vee L R(y) \phi_{x^{r}} \vee L R(y) \phi_{x^{r}}=L R(x) \phi_{y^{r}} \vee L R(y) \phi_{x^{r}}$ and $L R(y) \phi_{x^{r}} \equiv 0 \Longrightarrow L R(x) \phi_{y^{r}} \vee L R(y) \phi_{x^{r}} \equiv L R(x) \phi_{y^{r}} \equiv 0$.

THEOREM 3.4. Let $S$ be a right Baer semigroup and $\phi: S \rightarrow T$ an $R A P$ homomorphism. Then the equivalence relation $\equiv$ induced on $\mathscr{L}(S)$ by $\phi$ (recall $L R(x) \equiv L R(y)$ iff $L R(x \phi)=L R(y \phi)$ ) is an S-compatible join congruence.

Proof. S-compatibility was proven in Theorem 2.7. By Lemma 3.3 it is sufficient to show that $L R(x) \vee L R(z)=L R(y) \vee L R(z)$ and $L R(z \phi)=0 \Rightarrow L R(x \phi)=L R(y \dot{\phi})$. Now $L R(z \phi)=0$ means that $R(z \dot{\phi})=$ $\left(z^{r} \phi\right) T=T$, so $1 \phi=z^{r} \phi 1 \phi=z^{r} \phi$. Since $L R\left(x z^{r}\right)=(L R(x) \vee L R(z)) \phi_{z^{r}}=$ $(L R(y) \vee L R(z)) \phi_{z^{r}}=L R\left(y z^{r}\right)$, we have $L R(x \phi)=L R\left(x z^{r} \phi\right)=L R\left(y z^{r} \phi\right)=$ $L R(y \phi)$.

An S-compatible join congruence is determined by its kernel in the following manner.

THEOREM 3.5. If $S$ is a right Baer semigroup and $\equiv$ is an $S$ compatible join congruence on $\mathscr{L}(S)$, the following are equivalent.

(a) $L R(x) \equiv L R(y)$.

(b) $L R(x) \phi_{y^{r}} \vee L R(y) \phi_{x^{r}} \equiv 0$.

(c) There is an $L R(z) \equiv 0$ such that $L R(x) \vee L R(z)=L R(y) \vee L R(z)$.

Proof. $\quad(\mathrm{a}) \Rightarrow(\mathrm{b})$. If $L R(x) \equiv L R(y)$, then $L R(x) \phi_{y^{r}} \equiv L R(y) \phi_{y^{r}}=$ $0=L R(x) \phi_{x^{r}} \equiv L R(y) \phi_{x^{r}}$ and hence $L R(x) \phi_{y^{r}} \vee L R(y) \phi_{x^{r}} \equiv 0$. 
(b) $\Rightarrow$ (c). Follows from part (i ) of Lemma 3.2.

$(\mathrm{c}) \Rightarrow$ (a). Follows from Lemma 3.3.

CoRollary 3.6. An S-compatible join congruence $\equiv$ has the property that, for each $z \in S$, any choice of $z^{r}$ gives $L R(x) \equiv 0 \Rightarrow L R(x) \dot{\phi}_{z^{r}}^{+} \equiv$ $0 \phi_{z^{r}}^{+}$for all $x \in S$.

Proof. Since a join congruence is ordered, it is sufficient by Lemma 2.6 to show that $L R\left(x z^{r}\right) \equiv 0 \Rightarrow[L R(x)] \leqq[L R(z)]$. Since by part (iii) of Lemma 3.2 we have $L R(x) \vee L R(z) \vee L R\left(x z^{r}\right)=L R(z) \vee L R\left(x z^{r}\right)$, it follows from the theorem that when $L R\left(x z^{r}\right) \equiv 0, L R(x) \vee L R(z) \equiv L R(z)$. Since $\equiv$ is a join congruence, this says that $[L R(x)] \leqq[L R(z)]$.

THEOREM 3.7. If $S$ is a right Baer semigroup and $\equiv$ is an S-compatible join congruence on $\mathscr{L}(S)$, then the homomorphism $z \mapsto \Phi_{z}$ described in Lemma 2.9 is RAP.

Proof. We wish to show that $R\left(\Phi_{x}\right)=\Phi_{x} S^{\prime}$ or, in other words, that $\Phi_{x} \Phi_{y}=0 \Leftrightarrow \Phi_{y}=\Phi_{x} \Phi_{y}$. Notice that $\Phi_{x} \Phi_{y}=0 \Leftrightarrow[1] \Phi_{x} \Phi_{y}=[0] \Leftrightarrow$ $[L R(x y)]=[0] \Leftrightarrow L R(x y) \equiv 0$ and that $\Phi_{y}=\Phi_{x r} \Phi_{y} \Leftrightarrow L R(z y) \equiv L R\left(z x^{r} y\right)$ for all $z \in S$. Since it is clear that $\Phi_{y}=\Phi_{x r} \Phi_{y} \Rightarrow \Phi_{x} \Phi_{y}=0$, we will be done if we can show that $L R(x y) \equiv 0 \Rightarrow L R(z y) \equiv L R\left(z x^{r} y\right)$ for all $z \in S$. Since $L R(z y) \vee L R(x y)=L R\left(z x^{r} y\right) \vee L R(x y)$ by part (ii) of Lemma $3.2, L R(x y) \equiv 0$ implies by Theorem 3.5 that $L R(z y) \equiv L R\left(z x^{r} y\right)$ for all $z \in S$.

Corollary 3.8. If $S$ is a right Baer semigroup, then any S-compatible join congruence $\equiv$ on $\mathscr{L}(S)$ is induced by an RAP homomorphism on $S$.

Proof. Since, by Corollary 3.6 , $\equiv$ has property (i) of Theorem 2.7, the proof of Theorem 2.10 applies and says that $\equiv$ is induced on $\mathscr{L}(S)$ by the homomorphism $z \mapsto \Phi_{z}$ on $S$. By Theorem 3.7, $z \mapsto \Phi_{z}$ is $R A P$.

Corollary 3.9. If $S$ is a right Baer semigroup, then every $S$ - and $S^{+}$-compatible join congruence on $\mathscr{L}(S)$ is induced by an $R A P$ and l-preserving homomorphism on $S$.

Proof. This follows from Corollary 3.8 and from Theorem 2.12 and its proof.

CoRollary 3.10. If $S$ is a left Baer semigroup, then any $\mathrm{S}^{+-}$ 
compatible meet congruence on $\mathscr{L}(S)$ is induced by an LAP homomorphism on $S$.

Proof. This is the dual of Corollary 3.8. (See the remarks preceding Theorem 2.12.)

CoRollary 3.11 (Thorne). If $S$ is a Baer semigroup, then every $S$ - and $S^{+}$-compatible congruence on $\mathscr{L}(S)$ is induced by an AP homomorphism on $S$.

Proof. This follows from Corollaries 3.8 and 3.10 and from Theorem 2.12 and its proof.

\section{Kernels of S-compatible join congruences.}

THeOREM 4.1. Let $I$ be an ideal of a join semilattice $L=\mathscr{L}(S)$, $S$ a right Baer semigroup. The following are equivalent.

(a) I is the kernel of an S-compatible join congruence.

(b) $I \phi_{z} \subseteq I$ for each $z \in S$.

Proof. (a) $\Rightarrow(\mathrm{b})$. If $L R(x) \in I$, then $L R(x) \equiv 0$ and by $S$-compatibility $L R(x) \phi_{z} \equiv 0 \phi_{z}=0$, i.e., $L R(x) \phi_{z} \in I$.

(b) $\Rightarrow$ (a). Suppose $I \phi_{z} \subseteq I$ for each $z \in S$. Define $L R(x) \equiv L R(y)$ iff $L R(x) \vee L R(w)=L R(y) \vee L R(w)$ for some $L R(w) \in I$. It is easy to see that $\equiv$ is a join congruence. If $L R(x) \equiv L R(y)$, then $L R(x) \vee$ $L R(w)=L R(y) \vee L R(w)$ with $L R(w) \in I$ and since $\phi_{z}$, being a residuated map, preserves join we have $L R(x) \phi_{z} \vee L P(w) \phi_{z}=L R(y) \phi_{z} \vee L R(w) \phi_{z}$. Since $L R(w) \phi_{z} \in I$ it follows that $L R(x) \phi_{z} \equiv L R(y) \phi_{z}$. Clearly $\equiv$ has $I$ as its kernel.

LEMMA 4.2. In any semigroup $S$ with 0 , if $R(w)$ is a two-sided ideal, for some $w \in S$, then $L R(w)$ is a two-sided ideal. Hence, if $S$ is a pre-Baer semigroup, $L R(w)$ is two-sided if and only if $R(w)$ is twosided.

Proof. Suppose $R(w)$ is two-sided. $L R(w)$ is already a left ideal so we must show that it is a right ideal. Let $x \in L R(w), y \in S$, and $z \in R(w)$. We need $x y z=0$. But $y z \in R(w)$ since $R(w)$ is two-sided and hence $x y z=0$. The second assertion follows from the first and its dual.

Theorem 4.1 characterized kernels of S-compatible join congruences. We now look at principal ideals which occur as kernels of $S$-compatible join congruences. 
THEOREM 4.3. Let $S$ be a right Baer semigroup. The following are equivalent.

(a) $[0, L R(w)]$ is the kernel of an S-compatible join congruence on $\mathscr{L}(S)$.

(b) $L R(w)$ is the kernel of an RAP homomorphism on $S$.

(c) $L R(w) \phi_{x} \subseteq L R(w)$ for all $x \in S$.

(d) $x w^{r}=w^{r} x w^{r}$ for all $x \in S$ and for any choice of $w^{r}$.

(e) $L R(w)$ is a two-sided ideal.

(f) $R(w)$ is a two-sided ideal.

Proof. (a) $\Leftrightarrow(b)$. Since every RAP homomorphism $\phi$ on $S$ induces an $S$-compatible join congruence $\equiv$ on $\mathscr{L}(S)$ by the rule $L R(x) \equiv L R(y)$ iff $L R(x \phi)=L R(y \phi)$ and since every $S$-compatible join congruence arises in this manner for some $\phi$, it suffices to notice that $x \in \operatorname{ker} \phi \Leftrightarrow x \phi=$ $0 \Leftrightarrow L R(x \phi)=0 \Leftrightarrow L R(x) \equiv 0$.

(a) $\Leftrightarrow(\mathrm{c})$. Use Theorem 4.1.

(e) $\Leftrightarrow(f)$. Use Lemma 3.2.

$(d) \Leftrightarrow(f)$. This follows from the dual of Theorem 1 of [1].

(b) $\Rightarrow(\mathrm{e})$. This is obvious.

(d) $\Rightarrow$ (b). $\quad x \mapsto x w^{r}$ is a homomorphism of $S$ onto $S w^{r}$ and it is $R A P$ since $y w^{r} \in R\left(x w^{r}\right) \Leftrightarrow x w^{r} y w^{r}=0 \Leftrightarrow y w^{r}=w^{r} y w^{r}=x^{r} w^{r} y w^{r} \Leftrightarrow y w^{r} \epsilon$ $\left(x^{r} w^{r}\right)\left(S w^{r}\right)=(R(x)) w^{r}$.

REMARK. By Theorem 2.11, the kernel of an S-compatible join congruence $\equiv$ is a principal ideal if and only if $\equiv$ is residuated in the sense that the canonical join homomorphism taking $\mathscr{L}(S)$ onto $\mathscr{L}(S) / \equiv$ is a residuated map.

In light of Theorem 4.1 we make the following definition.

Definition 4.4. An ideal $I$ of a join semilattice $L=\mathscr{L}(S), S$ a right Baer semigroup, is called $S$-compatible in case $I \phi_{z} \subseteq I$ for all $z \in S$.

THEOREM 4.5. Let $S$ be a right Baer semigroup and let $L=\mathscr{L}(S)$. The set $I_{S}(L)$ of $S$-compatible ideals of $L$ forms a subcomplete sublattice of $I(L)$, the lattice of ideals of $L . I_{S}(L)$ is isomorphic to the lattice of S-compatible join congruences on $\mathscr{L}(S)$.

Proof. If $\left\{I_{i}\right\}$ is a family of $S$-compatible ideals of $\mathscr{L}(S)$ it is clear that $\bigcap_{i}\left\{I_{i}\right\}$ is an $S$-compatible ideal. Suppose $L R(x) \in \mathrm{V}_{i}\left\{I_{i}\right\}$. Then there exist

$$
L R\left(y_{1}\right) \in I_{i_{1}}, L R\left(y_{2}\right) \in I_{i_{2}}, \cdots, L R\left(y_{n}\right) \in I_{i_{n}}
$$


such that

$$
L R(x) \subseteq L R\left(y_{1}\right) \vee L R\left(y_{2}\right) \vee \cdots \vee L R\left(y_{n}\right)
$$

Hence

$$
\begin{aligned}
L R(x) \phi_{z} & \leqq\left(L R\left(y_{1}\right) \vee L R\left(y_{2}\right) \vee \cdots \vee L R\left(y_{n}\right)\right) \phi_{z} \\
& =L R\left(y_{1}\right) \phi_{z} \vee L R\left(y_{2}\right) \phi_{z} \vee \cdots \vee L R\left(y_{n}\right) \phi_{z}
\end{aligned}
$$

and since $L R\left(y_{k}\right) \phi_{z} \subseteq I_{i_{k}}(k=1,2, \cdots, n)$ we have $L R(x) \dot{\phi}_{z} \in \mathrm{V}_{i}\left\{I_{i}\right\}$. Thus $\mathrm{V}_{i}\left\{I_{i}\right\}$ is $S$-compatible and we have proven the first part of the theorem. Now, if $I \in I_{S}(L)$ let $\Theta_{I}$ denote the unique $S$-compatible join congruence with kernel $I$. In light of Theorem 3.5 it is clear that $I \leqq J \Leftrightarrow \Theta_{I} \subseteq \Theta_{J}$.

THEOREM 4.6. Let $S$ be a right Baer semigroup in which, for each $x \in S, L R\left(x^{l}\right)=L R\left(x^{l} x^{l}\right)$ for some choice of $x^{l}$. Then $I_{S}(L)$ is distributive and obeys the following infinite distributive law:

$$
I \cap\left(\mathrm{V}_{i}\left\{J_{i}\right)\right\}=\mathbf{V}_{i}\left\{I \cap J_{i}\right\} \text {. }
$$

Proof. It will suffice to show $I \cap\left(\mathrm{V}_{i}\left\{J_{i}\right\}\right) \subseteq \mathbf{V}_{i}\left\{I \cap J_{i}\right\}$. Suppose $L(x)=L R\left(x^{l}\right) \in I$ and $L R\left(x^{l}\right) \in \bigvee_{i}\left\{J_{i}\right\}$. Then $L R\left(x^{l}\right) \subseteq L R\left(y_{1}\right) \vee L R\left(y_{2}\right) \bigvee$ $\cdots \vee L R\left(y_{n}\right)$ where $L R\left(y_{k}\right) \in J_{i_{k}}(k=1,2, \cdots, n)$. Now $L R\left(x^{l}\right)=$ $L R\left(x^{l}\right) \phi_{x^{l}} \leqq L R\left(y_{1}\right) \phi_{x^{l}} \vee L R\left(y_{2}\right) \phi_{x l} \vee \cdots \vee L R\left(y_{n}\right) \dot{\phi}_{x^{l}}$. For $k=1,2, \cdots, n$ we have $L R\left(y_{k}\right) \dot{\phi}_{x l} \in J_{i_{k}}$ by $S$-compatibility and $L R\left(y_{h}\right) \phi_{x^{l}}=L R\left(y_{k} x_{l}\right) \subseteq$ $L R\left(x^{l}\right) \in I$. Thus $L R\left(y_{k}\right) \phi_{x^{l}} \in I \cap J_{i_{k}}$ for $k=1,2, \cdots, n$. Thus $L R\left(x^{l}\right) \in$ $\mathrm{V}_{i}\left\{I \cap J_{i}\right\}$

REMARK. Theorem 4.6 applies, in particular, when $S$ is a Baer semigroup. In that case $x^{l}$ is taken to be an idempotent generating $L(x)$. The $L R\left(x^{l}\right)=L R\left(x^{l} x^{l}\right)$ condition could also be taken care of by requiring, in the definition of pre-Baer semigroup, that $x^{r}$ and $x^{l}$ be idempotents. (It is pointed out in [2] that all our results involving pre-Baer semigroups remain valid if $x^{r}$ and $x^{l}$ are required to be idempotents.)

THEOREM 4.7. Let $S$ be a right Baer semigroup in which, for each $x \in S, L R\left(x^{l}\right)=L R\left(x^{l} x^{l}\right)$ for some choice of $x^{l}$. Let $L=\mathscr{L}(S) . \quad I_{S}(L)$ is pseudo complemented since it is complete and obeys the infinite distributive law of Theorem 4.6. If $I \in I_{S}(L)$, its pseudo complement $I^{*}$ is given by $I^{*}=\{L R(x) ; L R(x) \subseteq L(J)\}$, where $J$ is the kernel of any RAP homomorphism which induces the S-compatible join congruence with kernel $I$, i.e., $y \in J \Leftrightarrow L R(y) \in I$.

Proof. $I^{*}$ is an ideal since $L R(x), L R(y) \subseteq L(J) \Rightarrow J \subseteq R(x) \cap$ 
$R(y) \Rightarrow L R(x) \vee L R(y)=L(R(x) \cap R(y)) \subseteq L(J)$. Suppose $L R(x) \in I^{*}$ and $y \in S$. Then $z \in J \Rightarrow y z \in J \Rightarrow x y z=0 \Rightarrow x y \in L(J) \Rightarrow L R(x y) \subseteq L(J) \Rightarrow$ $L R(x) \phi_{y}=L R(x y) \in I^{*}$. Thus $I^{*}$ is $S$-compatible. Now suppose $L(x) \in$ $I \cap I^{*}$. Then $L(x)=L R\left(x^{l}\right) \in I \Rightarrow x^{l} \in J$ and $L R\left(x^{l}\right) \in I^{*} \Rightarrow x^{l} \in L R\left(x^{l}\right) \subseteq$ $L(J)$. Thus $x^{l} x^{l}=0$ and $L(x)=L R\left(x^{l}\right)=L R\left(x^{l} x^{l}\right)=0$. Therefore $I \cap I^{*}=0$. Finally, suppose $I \cap K=0$, with $K \in I_{S}(L)$. Let $L R(x) \in K$, $y \in J$. Then $L R(y) \in I \Rightarrow L R(x y) \subseteq L R(y) \in I$ and $L R(x) \in K \Rightarrow L R(x) \dot{\phi}_{y}=$ $L R(x y) \in K$. Thus $L R(x y) \in I \cap K=0 \Rightarrow x y=0 \Rightarrow x \in L(J) \Rightarrow L R(x) \subseteq$ $L(J) \Rightarrow L R(x) \in I^{*}$. Therefore $K \subseteq I^{*}$.

\section{REFERENCES}

1. T. S. Blyth and M. F. Janowitz, On decreasing Baer semigroups, Bull. Soc. Roy. Sci., Liège 38 (1969), 414-423.

2. C. S. Johnson, Jr., Semigroups coordinatizing posets and semilattices, J. London Math. Soc., to appear.

3. R. S. Pierce, Homomorphisms of semi-groups, Ann. of Math., 59 (1954), 287-291.

4. B. J. Thorne, A-P congruences on Baer semigroups, Pacific J. Math., 28 (1969). 681-698.

Recieved June 25, 1970. The results presented here were part of a thesis submitted to the University of Massachusetts. The research was supported by an NSF Traineeship. The author is grateful to Professor M. F. Janowitz for his generous help and guidance.

Bowling Green State University 



\title{
PACIFIC JOURNAL OF MATHEMATICS
}

\author{
EDITORS
}

\author{
H. SAMELSON \\ Stanford University \\ Stanford, California 94305 \\ C. R. HOBBY \\ University of Washington \\ Seattle, Washington 98105
}

J. DugundJI

Department of Mathematics

University of Southern California

Los Angeles, California 90007

RICHARD ARENS

University of California

Los Angeles, California 90024

\section{ASSOCIATE EDITORS}
E. F. BeCKenBaCH
B. H. NeUMANN
F. WOLF
K. Yoshida

\section{SUPPORTING INSTITUTIONS}

\author{
UNIVERSITY OF BRITISH COLUMBIA \\ CALIFORNIA INSTITUTE OF TECHNOLOGY \\ UNIVERSITY OF CALIFORNIA \\ MONTANA STATE UNIVERSITY \\ UNIVERSITY OF NEVADA \\ NEW MEXICO STATE UNIVERSITY \\ OREGON STATE UNIVERSITY \\ UNIVERSITY OF OREGON \\ OSAKA UNIVERSITY
}

\author{
UNIVERSITY OF SOUTHERN CALIFORNIA \\ STANFORD UNIVERSITY \\ UNIVERSITY OF TOKYO \\ UNIVERSITY OF UTAH \\ WASHINGTON STATE UNIVERSITY \\ UNIVERSITY OF WASHINGTON \\ AMERICAN MATHEMATICAL SOCIETY \\ NAVAL WEAPONS CENTER
}

The Supporting Institutions listed above contribute to the cost of publication of this Journal, but they are not owners or publishers and have no responsibility for its content or policies.

Mathematical papers intended for publication in the Pacific Journal of Mathematics should be in typed form or offset-reproduced, (not dittoed), double spaced with large margins. Underline Greek letters in red, German in green, and script in blue. The first paragraph or two must be capable of being used separately as a synopsis of the entire paper. The editorial "we" must not be used in the synopsis, and items of the bibliography should not be cited there unless absolutely necessary, in which case they must be identified by author and Journal, rather than by item number. Manuscripts, in duplicate if possible, may be sent to any one of the four editors. Please classify according to the scheme of Math. Rev. Index to Vol. 39. All other communications to the editors should be addressed to the managing editor, Richard Arens, University of California, Los Angeles, California, 90024.

50 reprints are provided free for each article; additional copies may be obtained at cost in multiples of 50 .

The Pacific Journal of Mathematics is published monthly. Effective with Volume 16 the price per volume (3 numbers) is $\$ 8.00$; single issues, $\$ 3.00$. Special price for current issues to individual faculty members of supporting institutions and to individual members of the American Mathematical Society: $\$ 4.00$ per volume; single issues $\$ 1.50$. Back numbers are available.

Subscriptions, orders for back numbers, and changes of address should be sent to Pacific Journal of Mathematics, 103 Highland Boulevard, Berkeley, California, 94708.

PUBLISHED BY PACIFIC JOURNAL OF MATHEMATICS, A NON-PROFIT CORPORATION

Printed at Kokusai Bunken Insatsusha (International Academic Printing Co., Ltd.), 270, 3chome Totsuka-cho, Shinjuku-ku, Tokyo 160, Japan. 


\section{Pacific Journal of Mathematics}

\section{Vol. 39, No. $3 \quad$ July, 1971}

William O'Bannon Alltop, 5-designs in affine spaces ................... 547

B. G. Basmaji, Real-valued characters of metacyclic groups ................. 553

Miroslav Benda, On saturated reduced products....................... 557

J. T. Borrego, Haskell Cohen and Esmond Ernest Devun, Uniquely representable semigroups. II.......................................

George Lee Cain Jr. and Mohammed Zuhair Zaki Nashed, Fixed points and stability for a sum of two operators in locally convex spaces ....................

Donald Richard Chalice, Restrictions of Banach function spaces ...............

Eugene Frank Cornelius, Jr., A generalization of separable groups ..............

Joel L. Cunningham, Primes in products of rings ......................

Robert Alan Morris, On the Brauer group of $Z$.

593

603

615

David Earl Dobbs, Amitsur cohomology of algebraic number rings ...............

Charles F. Dunkl and Donald Edward Ramirez, Fourier-Stieltjes transforms and

weakly almost periodic functionals for compact groups ...................

Hicham Fakhoury, Structures uniformes faibles sur une classe de cônes et

d'ensembles convexes ......................................

Leslie R. Fletcher, A note on C $\theta \theta$-groups.

Humphrey Sek-Ching Fong and Louis Sucheston, On the ratio ergodic theorem for

semi-groups............................................

James Arthur Gerhard, Subdirectly irreducible idempotent semigroups ...........

Thomas Eric Hall, Orthodox semigroups.....................

Marcel Herzog, $C \theta \theta$-groups involving no Suzuki groups ..........

669

687

John Walter Hinrichsen, Concerning web-like continua ..........

691

Frank Norris Huggins, A generalization of a theorem of F. Riesz.

695

Carlos Johnson, Jr., On certain poset and semilattice homomorphisms

703

Alan Leslie Lambert, Strictly cyclic operator algebras ...........

717

Howard Wilson Lambert, Planar surfaces in knot manifolds . . .

727

Robert Allen McCoy, Groups of homeomorphisms of normed linear spaces ....... 735

T. S. Nanjundiah, Refinements of Wallis's estimate and their generalizations ...... 745

Roger David Nussbaum, A geometric approach to the fixed point index .......... 751

John Emanuel de Pillis, Convexity properties of a generalized numerical range .... 767

Donald C. Ramsey, Generating monomials for finite semigroups ....

783

William T. Reid, A disconjugacy criterion for higher order linear vector differential equations...

Roger Allen Wiegand, Modules over universal regular rings...

Kung-Wei Yang, Compact functors in categories of non-archimedean Banach

spaces.

R. Grant Woods, Correction to: "Co-absolutes of remainders of Stone-Čech compactifications".

Ronald Owen Fulp, Correction to: "Tensor and torsion products of

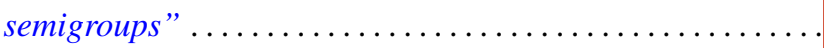

Bruce Alan Barnes, Correction to: "Banach algebras which are ideals in a banach 